\title{
What big data could achieve in Scotland
}

KE Walesby ${ }^{1}$, JK Harrison ${ }^{2}$, TC Russ ${ }^{3}$

Declaration of interests: No conflict of interests declared

\author{
Correspondence to: \\ KE Walesby \\ Alzheimer Scotland \\ Dementia Research Centre \\ 7 George Square \\ University of Edinburgh \\ Edinburgh EH8 9JZ \\ UK
}

Email:

katherine.walesby@ed.ac.uk

\section{What are big data?}

The term 'big data' is now frequently used in medicine. But what does this really mean and how is it relevant to the clinician as well as researcher? The background of this increasingly popular term is discussed here; what it means in clinical practice, for patients and for research; and why Scotland is poised to take advantage of this big data revolution.

There is no universal agreement on the meaning of big data. Indeed to many the term is vague, ill-defined and confusing. It generally refers to large or complex data, often requiring terabytes or petabytes of storage. It might refer to large amounts of information at a population, regional or local level or span different geographical areas. To put this in context, a terabyte could hold 1,000 copies of the Encyclopaedia Britannica and a petabyte 20 million four-door filing cabinets of information. ${ }^{1}$

Traditionally, the term 'big data' has been linked to the explosion in the ability to capture and store data generated by the use of electronic devices in daily life. However, it is increasingly recognised that it also describes the large volume of patient information collected in everyday health and social care practice. Less well recognised is that using so-called big data for clinical improvement has a much longer history. In 1839, William Farr (a British epidemiologist) realised the benefits of routinely collected data when he established a system for recording the causes of death. This allowed mortality rates to be compared for the first time and assisted in improving public health.
As clinicians and practitioners, we are constantly using or generating big data, often without awareness. Every patient admitted, discharged, reviewed in clinic or collecting a prescription generates valuable data which can be harnessed for improvements in care. One benefit of big data in medicine is that it is from real people (patients) using the NHS rather than the rarefied populations that take part in clinical trials.

Often, the power of big data is harnessed by data linkage. Data linkage is the process of combing datasets to ensure information on an individual is linked to several different outcomes (e.g. medications prescribed, number of and reason for hospital admissions). The concept of data linkage is not a new phenomenon. Throughout the 20th century, data linkage (or record linkage) has become an important aspect of public health practice. In 1946, Halbert Dunn (Chief, US National Office of Vital Statistics) described linking birth and death records:

Each person in the world creates a Book of Life. This Book starts with birth and ends with death. Its pages are made up of the records of the principal events in life. Record linkage is the name given to the process of assembling the pages of this Book, into a volume.

Health informatics is a term that encompasses using data linkage to create large electronic datasets to improve the health and care of patients and the public.

\section{Relevance to all - not just research}

Big data are not only relevant to researchers. The implications for practising clinicians, patients and researchers of

${ }^{1}$ Clinical Research Fellow, Geriatric Medicine, Alzheimer Scotland Dementia Research Centre and Centre for Cognitive Ageing and Cognitive Epidemiology, University of Edinburgh, UK and Specialist Registrar Geriatric and General Medicine, NHS Tayside; ${ }^{2} \mathrm{Clinical}$ Research Fellow, Geriatric Medicine, Alzheimer Scotland Dementia Research Centre and Centre for Cognitive Ageing and Cognitive Epidemiology, University of Edinburgh, UK; ${ }^{3} \mathrm{Co}$-Director, Alzheimer Scotland Dementia Research Centre, University of Edinburgh, UK; Centre for Cognitive Ageing and Cognitive Epidemiology, University of Edinburgh, UK, and Centre for Dementia Prevention, University of Edinburgh 
understanding healthcare, advice on healthcare policy and designing services is significant. In a digital world we are surrounded by data. Within medicine, data sometimes seem separate from our clinical job, and yet they are an integral part of delivering patient care and developing patient services. Since their introduction, electronic health records have collected vast amounts of information on the patient, the healthcare provider and the delivery of healthcare services. It is through these data that resources for clinical work can be allocated or a greater understanding of our patients' diseases or risk factors at a local, regional or national level can be gathered to improve patient care.

Everyone working in the NHS is by default involved with big data generation for current and future research and the wellbeing of patients, whether they are researchers or not. Therefore we all have a duty to understand how the recording of our patient-clinician interactions and diagnoses can influence the veracity of the big data that are later analysed, as well as how we can all realise the potential of big data in our individual specialties.

An example of big data in clinical practice is the Scottish Care Information Diabetes Collaboration (SCI-Diabetes), established in 2002. ${ }^{2}$ Real-time clinical information is collected in a fully integrated and shared electronic patient record by clinicians. It crosses the primary-secondary care interface and tracks information on all 300,000 patients with diabetes in Scotland. The detailed data gathered provide valuable insights into the diabetes community, including trends in treatment and clinical outcomes.

The Farr institute has highlighted 100 ways of using data to make lives better in their case examples, many using Scottish data. ${ }^{3}$ A powerful example from Scotland highlights the key role clinicians have in ensuring the accuracy of current diagnoses and past medical history recorded on electronic health records. ${ }^{4}$ A team at the Universities of Stirling and Glasgow and NHS Information Services Division Edinburgh looked at suicides linked with hospital records. A quarter of suicides were people who died within three months of their last hospital (general and psychiatric) discharges. Only 14\% discharged had a mental illness recorded during their last hospital visit despite one in five having a history of previous mental illness. ${ }^{4,5}$ This study highlights gaps in the current management and follow-up of these individuals and helps identify areas for targeted training and intervention to address this problem. These findings come from everyday practice but it is only by analysing these data can we find ways to prevent similar failures happening again. This highlights the crucial role all frontline staff have in contributing to the veracity of big health data.

The Twitter hashtag coined by the Farr institute is \#DataSavesLives. This exemplifies the Farr Institute's aim of highlighting how data can save lives by using health data in research to give new understanding and insights, which benefits patients and improves public health.

\section{Big data in Scotland}

Procedures for storing and accessing big data vary internationally and within the four nations of the UK. Some countries legislate for the use of data (e.g. Care Act 2014, England and Wales) and linking data sets, and others have a more pragmatic approach (e.g. Scotland).

A key feature to facilitate the linking of big datasets is to have a universal identifier present across all datasets, known as a unique identifier. In Scotland, the Community Hospital Index $(\mathrm{CHI})$ is a unique identifier which includes the person's date of birth (the first 6 digits) followed by a unique four digit ending (which indicates region of birth [or first registration] and gender). The decision in the 1970s for every person registered with a GP in Scotland to be allocated this unique identifying number from the $\mathrm{CHI}$ (centrally maintained) register was invaluable. The Scottish Record Linkage System, using the $\mathrm{CHI}$, started in the late 1980s. Compared with the NHS number, the $\mathrm{CHI}$ number is a person-specific identifier and must be used in every hospital or GP episode. Although the NHS number is a unique identifier, it was not primarily used for linking data. As it does not contain the patient's date of birth it is harder to ensure matched records belong to the same person. The $\mathrm{CHI}$ system makes this process much more efficient as it makes access to routine clinical data and health episodes for research easier and less expensive.

One of the most striking recent examples of using big data in Scotland is the evaluation of the effects of the smoking legislation introduced in $2006 .{ }^{6}$ Researchers were able to evaluate trends in hospital admissions to determine the impact of this national policy on the health of Scotland's citizens. ${ }^{7,8}$ Without using routinely collected big data, this evidence would have been more difficult, and expensive, to collect, relying on bespoke data collection.

Through this unique identifier, Scotland has created the Scottish Longitudinal Study, which incorporates census data linked to health records and education data. ${ }^{9}$ This is one of the world's largest administrative datasets. It demonstrates the feasibility of linking multiple data (health, demographic and socio-economic). This work is only possible due to the establishment of key centres in Scotland to ensure data linkage conforms to the key principles and governance of data linkage. These centres have developed over time as the recognition of the power of data linkage in improving healthcare and research is growing (Box 1).

\section{Characteristics of big data}

The three Vs of big data first described in 2001 by an American IT firm ${ }^{10}$ are still used and relevant now:

1. Volume - the scale/size of the data

2. Velocity - the speed at which it is generated

3. Variety - the varying forms the data is available in images, text 
Box 1 Overview of recent key centres in the development of big data in Scotland

\section{ScottisH Informatics Programme}

SHIP ${ }^{31}$ was initiated in 2009, and involving four universities and the Information Services Division (ISD) (part of NHS National Services Scotland), the principal source of statistics and healthcare services in Scotland. SHIP was the first organisation developing data linkage in Scotland to improve healthcare of patients and public. It is a Scotland-wide research platform for collating, managing, governing and analysing electronic patient records. This work has now moved to the Farr Institute.

\section{Farr institute of Health Informatics Research}

Dundee University was the original centre that led to the creation of the Farr Institute ${ }^{32}$ in Scotland. Following expansion, the Farr Institute is a well-recognised UKwide clinical research collaboration. Scotland now has seven (six universities and NHS Health Scotland) out of the 24 academic institutions and health partners across England, Scotland and Wales who are part of this UK-wide research collaboration. NHS Scotland is the principle health data custodian in Scotland.

\section{Administrative Data Research Network}

$\mathrm{ADRN}^{33}$ has centres in all four nations of the UK. The Administrative Data Research Centre (ADRC) for Scotland is led by the University of Edinburgh and incorporates seven major Scottish research centres. Administrative data refers to education, housing, transport etc. and that collected in specific surveys of health or care behaviours/ attributes, such as the Scottish Health Survey, which have been linked to health data. ${ }^{34}$

\section{Scottish Informatics and Linkage Collaboration}

SILC $^{35}$ is a collaboration of ADRN, The Farr Institute Scotland and The Urban Big Data Centre.

However, many have argued the recent addition of a fourth and fifth: Veracity (the accuracy of the data) and Value (allowing new insights and understanding). ${ }^{11,12}$

In medicine, this fourth point is of critical importance. If the data we have to analyse do not have sufficient accuracy, then the output and relevance to clinical practice will be questionable. When considering how big data can shape what we do now in healthcare, and may influence future work, it is important to consider the five Vs of big data.

\section{Challenges and complexities of big data}

There are many challenges in using big data to inform our understanding of health. These are partly exemplified by the rapid growth of data sets and constant generation of healthcare data from multiple sources. The challenges can be grouped into three key areas:

1. Data governance, including storage, transferring, sharing and privacy
2. Data generation, including capturing, curating, updating and accuracy

3. Data output, including analysis, querying large datasets and generating meaningful and reliable outputs

\section{Data governance}

There are numerous processes in place to safeguard the public and patients' interests. Navigating the path of research ethics and the variety of permissions required is never easy. ${ }^{13}$ These ensure that non-identifiable data are used and that it is for a worthy cause in the public's interest.

Public concern exists over use of patient data and the potential for commercialisation. ${ }^{14,15}$ Public engagement has emphasised that researchers must ensure that health and social care data linkage research does not risk identifying individuals, is secure and that it demonstrates benefits for patients and other service users or the public. ${ }^{16}$ Therefore, it is appropriate that there are strict guidelines, codes of conduct and governance that surround accessing patient data in Scotland. It is the role of everyone in the NHS to understand the governance and strict protocols in place that govern data linkage and analysis.

\section{Data generation}

It takes time to generate large datasets. Rarely are they complete and establishing the best method of linking datasets, to ensure the person with missing data is correctly linked to the related records, is vital. Advances in methodological developments of data linkage are rapid. ${ }^{17}$

One key challenge for analysing big health data is the ability to ascertain diseases through the accuracy of clinical coding. Diagnoses recorded by clinicians are coded according to the ICD-10 code of diseases ${ }^{18}$ Researchers are mindful of ensuring these diagnoses are accurate when using big data sources, ${ }^{19}$ rather than individual patient assessment methods.

Data are only as good as what has been entered by the individual doctor or nurse and then translated or transcribed by the coders in medical records. This is even more important when looking at large volumes of data; the belief that larger numbers result in a more accurate picture is not necessarily true.

\section{Data output}

If all diagnoses are transcribed but the coding is incorrect, this could result in erroneous calculations of the prevalence of different diseases and, importantly, the reasons for admission to hospitals. This has far reaching consequences when we combine this incorrect information with the basis for funding services or driving forward health policy. We know from several articles that incorrect coding adds to this inadequate knowledge of people who use the NHS. ${ }^{20}$ In times of a cash-strapped and over-stretched NHS, having accurate data on who uses the NHS and the conditions they present 
Box 2 The Scottish Morbidity Record

SMR 01 data lists all admissions and day cases where an individual is admitted to an NHS general hospital in Scotland according to their ICD code. ${ }^{36}$

\section{What is recorded?}

While there may be more than one diagnosis or medical problem during an admission episode, these may not all be included when translated from the paper or electronic records to the SMR 01 data. Additionally, if the coders in medical records are busy then only the first diagnosis is entered.

This is of relevance clinically, ensuring the first diagnosis is the most accurate in reflecting the patient's clinical picture. It is also important when considering national rates of diseases and reasons for hospital admissions.

\section{National prevalence of diseases}

Understanding the number of people in Scotland recorded with dementia is dependent on those with dementia being diagnosed and then clinicians recording dementia in clinical records. However, dementia is not commonly the principal reason for an acute admission to hospital, so it may not feature on the discharge summary unless recorded in 'other diagnoses' section. Therefore, a researcher looking for a disease such as dementia (rarely recorded as a primary reason for admission) may believe there are fewer people with a diagnosis of dementia using hospital admission data alone. We could draw the conclusion that those with dementia admitted to hospital, from the SMR 01 records, could be a far lower prevalence than would be accurate.

with is relevant to all of us. The Scottish Morbidity Record (SMR) in Box 2 illustrates how diagnoses are recorded and used for understanding diseases.

The accuracy of clinical coding must start from admission onwards. Discharge is an equally important time for ensuring accurate information to allow ICD codes for prevalence of diseases to be ascertained. All healthcare staff must be informed how the data they generate are used in order to help them understand the vital role of clinical coding and discharge correspondence to improve healthcare.

\section{Social care data}

Social care data can also provide a valuable opportunity to understand variations in patients' journeys. Thirty-two local authority areas in Scotland have responsibility for the provision of social care ${ }^{21}$ to the larger 14 regional health board areas. $^{22}$ Therefore, a health board will include residents from multiple local authorities. This distinction can manifest as variation in patient services available within the same ward. This includes access to home care services and care home funding. As social care support directly influences the ability to safely discharge patients, understanding the variation across Scotland is vital, particularly as our population ages and care needs increase. The Scottish Government has commissioned the Information Services Division to work with local authorities and NHS boards in the Health and Social Care Data Integration and Intelligence Project. ${ }^{23}$ A key aim is to develop linked, shared databases containing individuallevel anonymised health and social care data to evaluate interventions, policies and trends. ${ }^{23}$ Central analysis will allow comparisons between local authority areas. By identifying variation, regions can consider each other's services and learn from examples of good practice to improve outcomes, in keeping with a key strand (reducing unnecessary variation in practice and outcomes) of the Chief Medical Officer's report. ${ }^{24}$

The Scotland Social Care Survey is collecting information on home care usage, community alarms, telecare, and selfdirected support. ${ }^{25}$ The Care Home Census, collected since 2002 , is another source of social care data in Scotland. It provides aggregate data on care homes, places, residents, length of stay and individual-level data on long-stay admissions. ${ }^{26}$ Unlike the Social Care Survey (which produces a snapshot of use at one time), the annual Care Home Census aims to capture activity throughout the year; however, the completeness of these data has not been formally assessed. Both resources will have $\mathrm{CHI}$ added to help facilitate linkage with other routinely-collected data sources.

Analysis of English social care data suggests greater issues with data quality than those found in health data, although the authors propose some of this could be addressed by linking the two, maximising their usefulness. ${ }^{27}$ A pilot project in NHS Tayside found that differences in documentation, recording and variables meant the work was feasible but challenging and required joint working to understand organisational culture and practice. ${ }^{28}$ Building and maintaining crossorganisational relationships takes time. ${ }^{28}$

\section{The future}

Scotland is poised to take advantage of the big data revolution in healthcare due to the unique identifier $(\mathrm{CHI})$, one data custodian (NHS Scotland), and collaborative organisations for data linkage. Few countries have the ability to link high quality data of national coverage to allow patient-based analysis and follow up, and as the drive to integrate health and social care data increases this will provide a valuable additional resource to understand the patient journey.

Routinely collected health and social care data can be used to ensure practice is evidence-based, by examining in realtime the effects of changes at a local or national level on the outcomes of those directly affected. Comparison between areas to identify and explore variations allows targeted work to learn from and share good practice. Following the integration of health and social care in law, ${ }^{29}$ the impetus is there to deliver improvements to the lives of the people of Scotland through more effective joint working.

There is tremendous potential to improve practice and conduct relevant research using routinely-collected data to 


\section{What can clinicians do to improve the quality of healthcare big data?}

- Discharge letters - when listing several diagnoses linked with a patient's admission, consider the principal diagnosis that you want to be recorded as the first diagnosis. This may be the only diagnosis later used for establishing hospital admission data by disease within SMR data

- Death certificates - when issuing a death certificate, ensure that all relevant key medical diseases not linked with the primary cause of death are recorded in the secondary cause of death. This will allow more accurate national recording of prevalence of diseases at death

- $\mathrm{CHI}$ number - when documenting patient information, ensuring the $\mathrm{CHI}$ number (or appropriate unique identifier) is recorded on all patient records is key to facilitating record linkage. In many cases, where work is done electronically, this is automatically populated, but in situations where the clinician needs to complete records manually (e.g. handwritten prescriptions) it will allow a complete picture of the patient's journey to be recorded through data linkage help understand how to work towards delivering optimal care at home or in a homely setting, the goal outlined in both the Scottish Government's Vision 2020 and the Six Essential Actions to Improving Unscheduled Care..$^{30}$

\section{Funding}

KEW and JKH are supported by clinical research fellowships from Alzheimer Scotland and the University of Edinburgh Centre for Cognitive Ageing and Cognitive Epidemiology, part of the cross council Lifelong Health and Wellbeing Initiative (MR/ L501530/1). Funding from the Biotechnology and Biological Sciences Research Council (BBSRC) and Medical Research Council (MRC) is gratefully acknowledged. The funders played no part in the design or conduct of this paper.

\section{References}

1 What's a Byte? Megabytes, Gigabytes, Terabytes What are they? http://www.whatsabyte.com (accessed 18/1/17).

2 Scottish Care Information Diabetes Collaboration. http://www.scidiabetes.scot.nhs.uk (accessed 18/1/17).

3 The Farr Institute of Health Informatics Research. 100 Ways of Using Data to Make Lives Better. http://www.farrinstitute.org/ public-engagement-involvement/100-ways-of-using-data-to-makelives-better (accessed 18/1/17).

4 University of Glasgow/University of Stirling/NHS National Services Scotland/ISD Scotland. Can Data Be Used to Help Prevent Suicides? http://www.farrinstitute.org/wp-content/uploads/2016/10/11. Can-Data-Be-Used-To-Help-Prevent-Suicides-print-1.pdf (accessed 18/1/17).

5 Dougall N, Lambert P, Maxwell M et al. Deaths by suicide and their relationship with general and psychiatric hospital discharge: 30-year record linkage study. Br J Psychiatry 2014; 204: 267-73.

6 Haw SJ, Gruer L, Amos A, Currie C et al. Legislation on smoking in enclosed public places in Scotland: how will we evaluate the impact? J Public Health 2006; 28: 24-30.

7 Pell JP, Haw S, Cobbe S et al. Smoke-free legislation and hospitalizations for acute coronary syndrome. N Engl J Med 2008; 359: 482-91.

8 Mackay D, Haw S, Ayres JG et al. Smoke-free legislation and hospitalizations for childhood asthma. N Engl J Med 2010; 363: 1139-45.

9 Introduction to the Scottish Longitudinal Study. http://sls.Iscs. ac.uk/about (accessed 18/1/17).

10 Laney D. 3D Data Management: Controlling Data Volume, Velocity and Variety. 2001. http://blogs.gartner.com/doug-laney/ files/2012/01/ad949-3D-Data-Management-Controlling-DataVolume-Velocity-and-Variety.pdf (accessed 18/1/17).

11 IBM. Infographics \& Animations: The Four V's of Big Data. http:// www.ibmbigdatahub.com/infographic/four-vs-big-data (accessed 18/1/17).

12 Burbank D. The 5 V's of Big Data. Enterprise Architects. 2016. http://enterprisearchitects.com/the-5v-s-of-big-data (accessed $7 / 2 / 17)$
13 Brett CE, Deary IJ. Realising health data linkage from a researcher's perspective: following up the 6-Day Sample of the Scottish Mental Survey 1947. Longitudinal and Life Course Studies 2014; 5: 283-98.

14 National Data Guardian. National Data Guardian for Health and Care: Review of Data Security, Consent and Opt-Outs 2016. https:// www.gov.uk/government/uploads/system/uploads/attachment_ data/file/535024/data-security-review.PDF (accessed 18/1/17).

15 Carter P, Laurie GT, Dixon-Woods M. The social licence for research: why care.data ran into trouble. J Med Ethics 2015; 41: 404-9.

16 Scottish Government. Linking Social Care, Housing and Health Data: Social Care Clients' and Patients' Views. 2011. http://www.gov.scot/ Publications/2011/09/20085846/11 (accessed 8/1/16).

17 Harron K, Goldstein H, Dibben C, editors. Methodological Developments in Data Linkage. Chichester: John Wiley \& Sons, Ltd; 2016.

18 International Statistical Classification of Diseases and Related Health Problems. 10th Revision. 2016. http://apps.who.int/ classifications/icd10/browse/2016/en (accessed 18/1/17).

19 Woodfield R, Grant I, Sudlow CL. Accuracy of electronic health record data for identifying stroke cases in large-scale epidemiological studies: a systematic review from the UK Biobank Stroke Outcomes Group. PLoS One 2015; 10: e0140533.

20 Medford AR. Aberrant clinical coding: an underappreciated entity for NHS Trusts, clinicians and coders. J R Coll Physicians Edinb 2013; 43: 101-2.

21 Scottish Government. Scottish local authorities 2016. http://www. gov.scot/Topics/Government/local-government/localg/usefullinks (accessed 5/6/17).

22 Scottish Government. NHS Boards. http://www.gov.scot/Topics/ Health/NHS-Workforce/NHS-Boards (accessed 22/12/16).

23 NHS National Services Scotland/ISD Scotland. A Guide to Data to Support Health \& Social Care Partnerships in Joint Strategic Commissioning and Joint Strategic Needs Assessment. 2014. http://www.isdscotland.org/Health-Topics/Health-and-SocialCommunity-Care/docs/Guide-to-Data-to-Support-HSCPs-80ct2014FINAL.pdf (accessed 22/12/16). 
24 NHS Scotland. Chief Medical Officer's Annual Report 2014-15: RealisticMedicine.http://www.gov.scot/Resource/0049/00492520. pdf (accessed 28/1/16).

25 Scottish Government. Social Care Services, Scotland, 2016. http:/ www.gov.scot/Resource/0051/00510699.pdf (accessed 22/12/16).

26 Information Services Division Scotland. Care Home Census. Scottish Statistics on Adults Resident in Care Homes. 2015. http://www. isdscotland.org/Health-Topics/Health-and-Social-Community-Care/ Publications/2015-10-20/2015-10-20-CHCensus-Report.pdf? (accessed 20/10/15).

27 Kemm JR, Robinson J, Verne J. Social care data in England: what they tell us and what they do not tell us. Public Health 2010; 124: 265-8. http://www.sciencedirect.com/science/article/pii/ S003335061000065X

28 Witham MD, Frost H, McMurdo M et al. Construction of a linked health and social care database resource - lessons on process, content and culture. Inform Health Soc Care 2015; 40: 229-39.

29 Scottish Government. Integration of Health and Social Care. 2016. http://www.gov.scot/Topics/Health/Policy/Adult-HealthSocialCare-Integration
30 Scottish Government. 6 Essential Actions to Improving Unscheduled Care. http://www.gov.scot/Topics/Health/Quality-ImprovementPerformance/UnscheduledCare/6-Essential-Actions-To-ImprovingUnscheduled-Care (accessed 16/1/17).

31 ScottisH Informatics Programme (SHIP). http://www.scot-ship. ac.uk/index.html (accessed 18/1/17)

32 The Farr Institute of Health Informatics Research. http://www. farrinstitute.org (accessed 18/1/17).

33 Administrative Data Research Centre Scotland. https://adrn. ac.uk/about/network/scotland (accessed 18/1/17).

34 Hanlon P, Lawder R, Elders A et al. An analysis of the link between behavioural, biological and social risk factors and subsequent hospital admission in Scotland. J Public Health 2007; 29: 405-12.

35 Scottish Informatics and Linkage Collaboration. http://www. datalinkagescotland.co.uk (accessed 18/1/17).

36 ISD Scotland Data Dictionary. SMR01. http://www.ndc.scot.nhs. u k / D i c t i o n a r y - A - Z / D e fin it i o n s / i n d e x. asp?Search=S\&ID=997\&Title=SMR01 (accessed 18/1/17).

\section{The College Journal Prize}

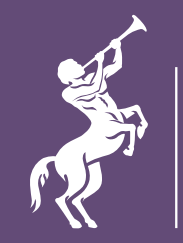

ROYAI

COLLEGE of

PHYSICIANS of

EDINBURGH

The College Journal Prize 2016, sponsored by the Senior Fellows' Club, was won by RT Tran and AJ Rankin (joint first authors) et al. for their paper 'Short runs of atrial arrhythmia and stroke risk: a European-wide online survey among stroke physicians and cardiologists'. This paper can be read in issue 2, 2016, http://www.rcpe.ac.uk/sites/default/files/jrcpe_46_2_tran.pdf

A prize of $£ 250$ will be awarded to the first-named (or corresponding) author of an original research paper on a clinical topic, deemed by a panel of judges to be the best paper by a doctorin-training (i.e. pre-consultant level) published in the Journal of the Royal College of Physicians of Edinburgh in issue 4, 2016 and issues 1, 2 and 3, 2017. The prize-winner will be invited to give a short oral presentation based on their paper at the Medical Trainees' Conference 2018.

Further details may be obtained from the Editorial Office, Royal College of Physicians of Edinburgh, 9 Queen Street, Edinburgh EH2 1JQ

Tel +44 (0)131 2473666 or email editorial@rcpe.ac.uk 